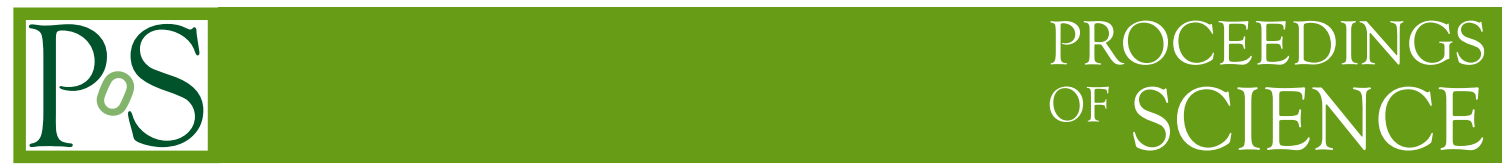

\title{
Measurement of inclusive production of light charged hadrons at BaBar
}

\section{Isabella GARZIA*}

SLAC and INFN-Sezione di Ferrara

E-mail: garzia@fe.infn.it

\begin{abstract}
Inclusive hadron production cross section in $e^{+} e^{-}$collisions shed light on fundamental questions of hadronization and fragmentation processes. We present measurements of inclusive spectra of charged pions, kaons, and protons (antiprotons) produced in $e^{+} e^{-}$collisions at the center-ofmass energy of $10.54 \mathrm{GeV}$. These results help test the scaling properties of the cross sections by comparison with previous measurements at higher center-of-mass energies and with theoretical predictions.
\end{abstract}

XXI International Workshop on Deep-Inelastic Scattering and Related Subject -DIS2013, 22-26 April 2013

Marseilles, France

\footnotetext{
* Speaker.
} 


\section{Introduction}

The production of hadrons from quark and gluon fragmentation in high-energy collisions is understood only qualitatively. The process $e^{+} e^{-} \rightarrow q \bar{q} \rightarrow$ hadrons can be described through three stages. First, the quark $(q)$ and antiquark $(\bar{q})$ fragment via radiation of gluons, each of which can radiate further gluons or split into a $q \bar{q}$ pair. This process is, in principle, calculable in perturbative QCD, but the difficulty of such calculations has limited them to low orders in $\alpha_{s}[1,2]$ or leading logs [3]. In the second stage, which is understood only qualitatively, the produced partons "hadronize", or transform into primary hadrons, which then decay into stable hadrons in the last stage. There are several phenomenological models used to reproduce the parton production stage, such as JETSET [4], HERWIG [5] and UCLA [6]. Each model contains a set of parameters used to describe the hadronization process, whose values have been tuned to reproduce data from $e^{+} e^{-}$ annihilation experiments. One fundamental test is the study of the scaling properties, which needs accurate inclusive measurement of hadron production also at low center-of-mass energies $\left(E_{C M}\right)$, since previous measurements performed by ARGUS [7] at the $E_{C M} \sim 10 \mathrm{GeV}$ were not able to cover the full kinematic range.

We present measurements of cross sections for inclusive production of charged pions, kaons, and protons (antiprotons) at the center-of-mass $(\mathrm{CM})$ energy $\sqrt{s}=10.54 \mathrm{GeV}$, using a relatively small sample of data from the BaBar experiment at the PEP-II B factory at the SLAC National Accelerator Laboratory. BaBar data are consistent with ARGUS results and extend the coverage up to highest momenta with an accuracy at the few-percent level.

\section{Cross section measurements}

The data used in this analysis [8] correspond to an integrated luminosity of $0.91 \mathrm{fb}^{-1}$ at a $\mathrm{CM}$ energy of $10.54 \mathrm{GeV}$. In parallel, $3.6 f b^{-1}$ of data recorded at the $\Upsilon(4 S)$ resonance $\left(E_{C M}=10.58\right.$ $\mathrm{GeV}$ ) are also analyzed. The latter sample provides independent, stringent systematic checks, and the combined samples provide data-driven calibrations of the tracking and particle identification performance.

We select good reconstructed charged tracks from multi-hadronic events, coming from the primary interaction point, in order to minimize backgrounds from other physics process. The identification of charged tracks as pions, kaons or protons is performed using an algorithm that combines the momentum and ionization energy loss $(d E / d x)$ measured in the Drift Chamber (DCH) [9] and the velocity measured via the Cherenkov angle in the DIRC detector [9]. The $d E / d x$ measurements from the DCH provide very good separation between low momentum particles, i.e. between $K^{ \pm}$ and $\pi^{ \pm}\left(p / \bar{p}\right.$ and $\left.K^{ \pm}\right)$below $0.5(0.8) \mathrm{GeV} / \mathrm{c}$, while the Cherenkov angle measurement from the DIRC provides a very good separation between particles with momentum between the Cherenkov threshold and the resolution limit of about $4 \mathrm{GeV} / \mathrm{c}$ for $\pi^{ \pm}$vs. $K^{ \pm}$and $6.5 \mathrm{GeV} / \mathrm{c}$ for $K^{ \pm}$vs. $p / \bar{p}$. The performance of our hadron identification is described in terms of a momentum-dependent efficiency matrix $\mathbf{E}$, where each element $E_{i j}$ represents the probability that a selected track from the true $i$-hadron is identified as a $j$-hadron, with $i, j=\pi, K p$. We calibrate the efficiency matrix using samples of tracks with know hadron content and characteristics as similar as possible to our selected tracks, and we derive corrections to the simulated $E_{i j}$ that vary smoothly with momentum 
and polar angle in the laboratory frame. We extract the raw differential cross section per selected events per unit momentum $p_{\text {lab }}$ in the laboratory frame $\left(\left(1 / N_{\text {evts }}\right) d n_{\pi, K, p} / d p_{\text {lab }}\right)$ from our sample of identified pions, kaons and protons using the corrected efficiency matrix. Then we subtract backgrounds and correct the spectra for the effects of detector efficiency and resolution, and the event selection procedure. Finally, we transform the cross sections into the $e^{+} e^{-} \mathrm{CM}$ frame in order to obtain the corrected differential cross sections $\left(1 / N_{\text {evts }}\right) d n_{\pi, K, p} / d p$. All possible systematic effects are carefully studied, and the main contributions come from particle identification, tracking efficiency, and background estimate. The statistical uncertainties are much smaller than the systematic uncertainties, with few exceptions at the highest momentum, and lower momentum for proton.

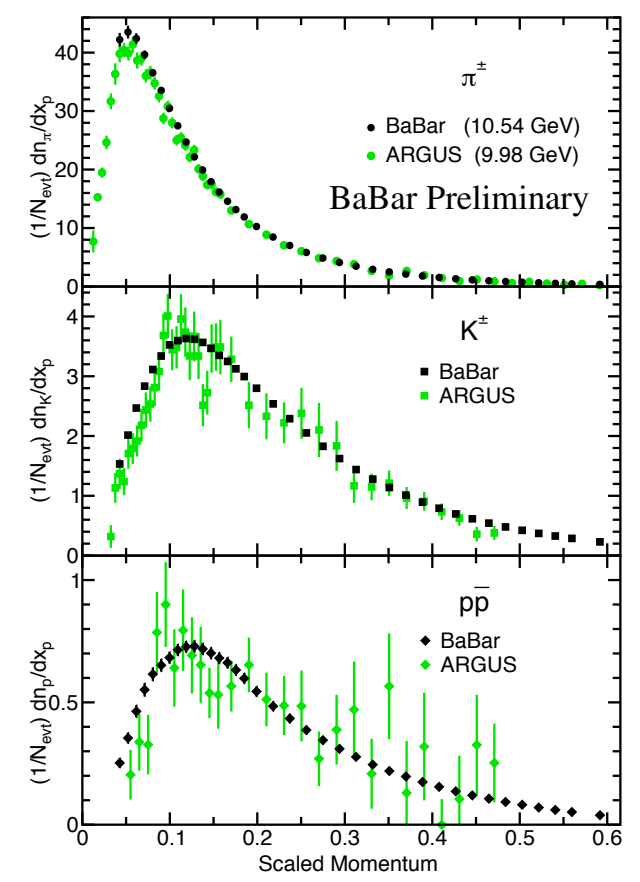

Figure 1: Measured differential cross sections for $\pi^{ \pm}$(top), $K^{ \pm}$(middle), and $p / \bar{p}$ (bottom), compared with previous results from ARGUS [7].

Figure 1 shows the differential cross sections for $\pi, K$, and $p / \bar{p}$ as a function of scaled momentum $x_{p}=2 p_{C M} / E_{C M}$ (black points). Previous measurements from the ARGUS experiment obtained at the slightly lower $E_{C M}=9.98 \mathrm{GeV}$ [7] are also reported. Both statistical and systematic errors are included. We observe that ARGUS $\pi^{+}$and $K^{ \pm}$data extend to lower $x_{p}$ values, whereas our results are very precise and extend up to $x_{p}=1$, so that most of the momentum range is covered by the two experiments. For $x_{p}>0.1$ the two data sets are consistent. For lower values, ARGUS data are systematically below BaBar data, as might be expected from a mass-driven scaling violation.

\subsection{Scaling properties}

In Fig. 2, we compare our results with those obtained at higher energies from TASSO at 34 $\mathrm{GeV}$ [10] and SLD at $91.2 \mathrm{GeV}$ [11]. The nearly full kinematic range coverage and the high precision of BaBar and SLD data, allow to study scaling properties of hadronization and to test 
predictions of different models of hadronization. Since QCD should be scale invariant, scaling violation effects are expected at low $x_{p}$ due to the mass of hadrons, while at higher momentum substantial scaling violation is expected because of the running of the strong coupling $\alpha_{s}$. This
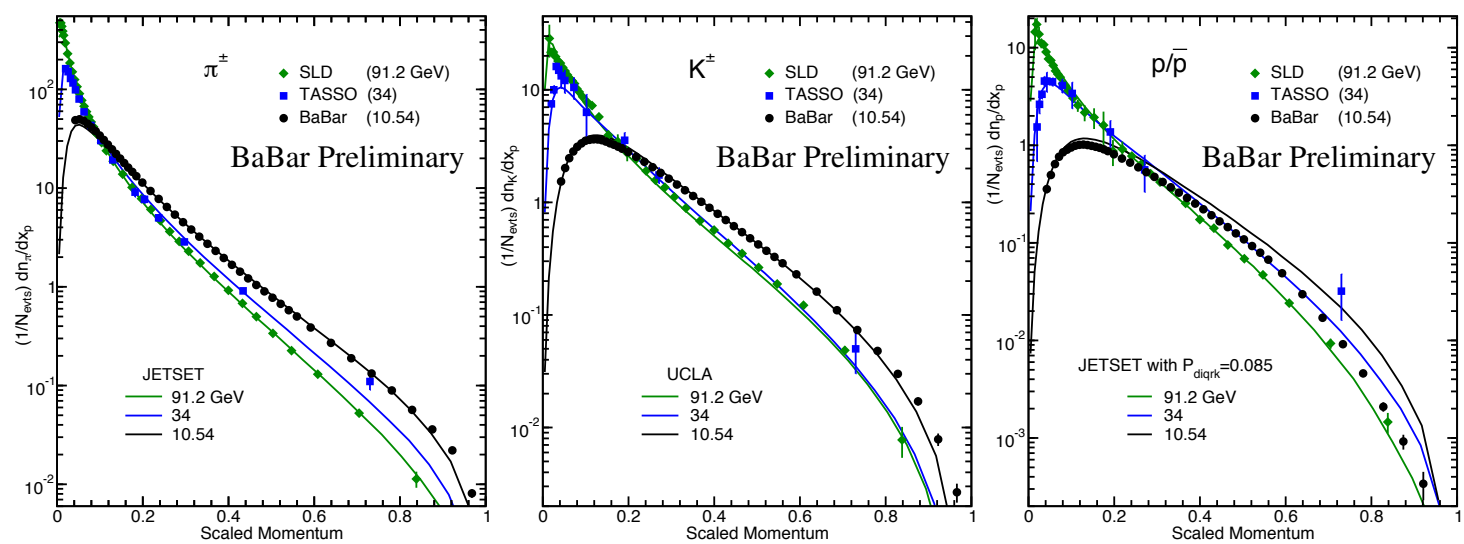

Figure 2: Differential cross sections for pions (left), kaons (middle), and proton (right) measured at three different $\mathrm{CM}$ energies: $10.54 \mathrm{GeV}$ (BABAR, black points), $34 \mathrm{GeV}$ (TASSO, blue squares), and $91.2 \mathrm{GeV}$ (SLD, green diamonds). Predictions by JETSET (for pions and protons) and UCLA (for kaons) are superimposed to the data point following the same color code.

behavior for pions is well reproduced by most of the hadronization models, and in Fig. 2(left) the JETSET prediction is shown. It provides a good description of all the three data sets using the default parameters ${ }^{1}$, and describes the high- $x_{p}$ scaling violation well. The middle plot in Fig. 2 shows the cross sections for $K^{ \pm}$. In this case, the different flavor composition of the three samples modified the expected scaling, and a difference of about $15 \%$ between models and data is observed. Finally, for $p / \bar{p}$ (Fig. 2(right)), the prediction for $10.54 \mathrm{GeV}$ is consistent with BaBar results for $x_{p}<0.07$, but it exceeds data point for higher $x_{p}$. In particular, no model predicts the correct scaling violation for protons, even though they describe well the properties for pions. These results will be used as inputs for tuning the simulation of fragmentation processes down to an energy of $10 \mathrm{GeV}$.

\subsection{Test of QCD predictions in the MLLA model}

These data can also be used to test some predictions of QCD in the Modified Leading Logarithm Approximation (MLLA) [3], combined with the ansatz of Local Parton-Hadron Duality (LPHD) [3]. For this purpose, it is convenient to plot our cross sections as a function of the variable $\xi=-\ln \left(x_{p}\right)$. MLLA predicts that a Gaussian function should provide a good description of these spectra around the peak position $\xi^{*}$, and that a slightly distorted Gaussian function should fit the data points over a wider range. Furthermore, the peak position $\xi^{*}$, should decrease exponentially with increasing hadron mass at a given $E_{C M}$. Figure 3 shows the distributions for $\pi^{ \pm}, K^{ \pm}$, and $p / \bar{p}$ with the results of the fits. The distorted Gaussian is able to describe the BaBar data at the few percent level over a large range of $\xi$. In addition, we find that $\xi_{\pi}^{*}$ is higher than $\xi_{K}^{*}$, in agreement

\footnotetext{
${ }^{1}$ The default parameters have been chosen based on previous data, mostly at the peak of the $Z_{0}$ boson, but including the ARGUS data.
} 

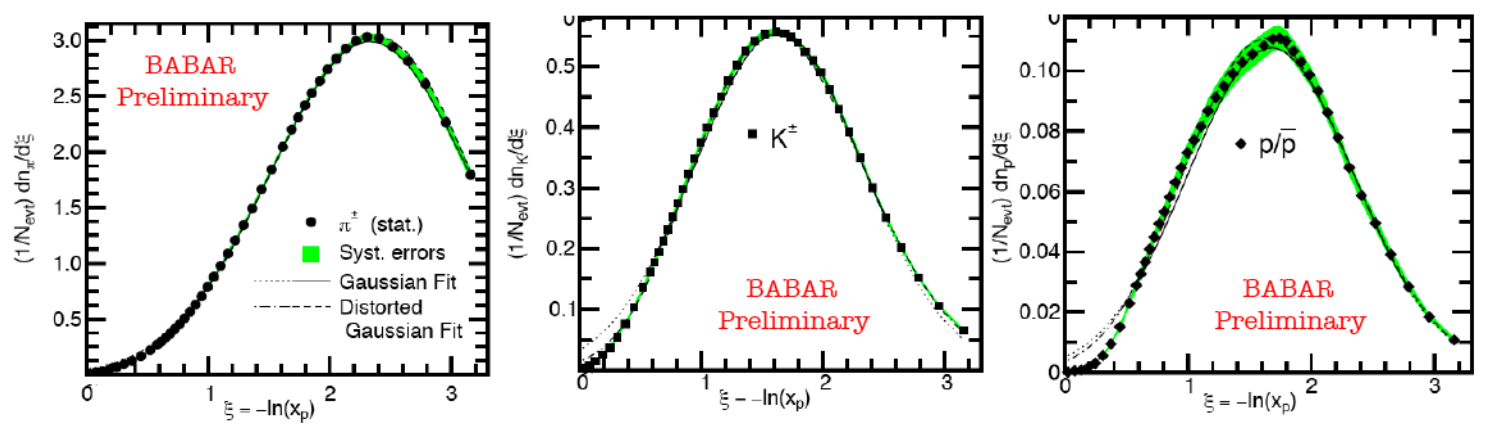

Figure 3: Differential cross sections vs. $\xi$ variable for pions (left), kaons (middle), and $p / \bar{p}$ (right). The error bar are statistical only, while the grey bands represent the systematic uncertainties. There are also shown the results of the Gaussian (solid line) and distorted Gaussian (dashed lines) fits.

with the predicted drop with hadron mass, but the $\xi_{p}^{*}$ is not lower than $\xi_{K}^{*}$ and thus inconsistent with the expected exponential decrease. This qualitative behavior is also observed at higher energies, where baryons and mesons appear to follow different trajectories, but measurements for more particles at $E_{C M} \sim 10 \mathrm{GeV}$ would be needed to draw any definite conclusion.

\section{Conclusions}

Preliminary precision measurements of the differential production cross section for charged pions, kaons, and protons have been performed at $\mathrm{BaBar}$ at $E_{C M}=10.54 \mathrm{GeV}$. They cover a wide range of $x_{p}$, and are the first measurements above $3 \mathrm{GeV} / c$ of momentum at $\sqrt{s} \sim 10 \mathrm{GeV}$. These data will be very valuable for a better understanding of the fragmentation process, and to study the scaling properties down to the energy of $10 \mathrm{GeV}$. In particular, the discrepancies between data and Monte Carlo predictions on scaling violation gives important experimental inputs for tuning models.

\section{References}

[1] See e.g. R.K. Ellis, D.A. Ross and A.E. Terrano, Nucl. Phys. B 178, 421 (1981).

[2] See e.g. S. Moretti, Phys. Lett. B 420, 367 (1998).

[3] Y.I. Azimov, Y.L. Dokshitzer, V.A. Khoze and S.I. Troian, Z. Phys. C 27, 65 (1985).

[4] T. Sjostrand, Comput. Phys. Commun. 82, 74 (1995).

[5] G. Corcella et al., JHEP 0101, 010 (2001); G. Marchesini et al., Comput. Phys. Commun. 67, 465 (1992).

[6] S. Chun and C. Buchanan, Phys. Rep. 292, 239 (1998).

[7] H. Albrecht et al. (ARGUS Collaboration), Z. Phys. C 44, 547 (1989).

[8] J.P. Lees et al., (BABAR Collaboration), arXiv:1306.2895 (2013).

[9] B. Aubert et al., (BABAR Collaboration), arXiv:1305.3560 (2013).

[10] W. Braunschweig et al., (TASSO Collaboration), Z. Phys. C 42, 189 (1989).

[11] K. Abe at al., (SLD Collaboration), Phys. Rev. D 59, 052001 (1999). 